\title{
Microwave-photonic networks based on single-mode multi-core optical fibers
}

\author{
Zbigniew Zakrzewski ${ }^{* 1}$ \\ ${ }^{1}$ Faculty of Telecommunications, Computer Science and Electrical Engineering, University of Technology and Life \\ Sciences, Al. Prof. S. Kaliskiego 7, 85-796 Bydgoszcz
}

Received October 10, 2013; accepted December 19, 2013; published December31, 2013

\begin{abstract}
In the paper, the proposition to construct a 9-core singlemode optical fiber TA-MCF (Trench Assisted - Multi-Core Fiber) has been presented. The nine-core TA-MCF can be used to connect multiantenna array modules to the radio-photonic modems located at the signal processing central. Optical networks for transporting radio signals, called RoF (Radio over Fiber) Networks, allow space distributing of the radio base-station eNodeB over a greater area. Multicore fibers make such solutions possible.
\end{abstract}

Microwave-photonic techniques implemented in the radio over fiber networks (RoF networks) [1] allow space disposal of antenna systems and radio resources. Radio signals can be provided from the central distribution radio signal point to individual distributed antenna modules, giving a high decorrelation coefficient of the beams involved to diversity or spatial multiplexing.

Thee group of modern optical telecommunication fibers based on silica $\mathrm{SiO}_{2}$ can be divided into multi-mode and single-mode optical fibers. Multi-mode fibers are characterized by guiding many modes that have a high coefficient of coupling between them, which strongly hampers their use in spatial multiplexing techniques, although does not prevent entirely [2-3]. Single-mode optical fibers SMF are characterized by guiding only one mode but in two orthogonal polarizations. In that optical waveguide, spatial modal multiplexing, cannot be implemented, excluding polarization.

Multi-core single-mode optical fibers MCF with builtin few cores in the cladding and single-core few-mode optical fibers FMF with an extended core diameter and a profiled index in the space of a few-mode core can be classified as the next generation of telecommunication optical fibers NG-OF.

An antenna array module, usually including 4 or 8 elements, is applied in modern antenna systems. It allows to create a radio connection with maximal spatial multiplexing SDM-MIMO (Spatial Division Multiplexing - Multiple Input Multiple Output) $4 \times 4$ or $8 \times 8$. In the case of implementing the antenna distributed technique over a large area, software defined radio SDR, and the smart cognitive radio resource management, there is a need for efficient delivery and removal of radio signals to/from the signal processing centers. It can be done by distributing radio signals in fiber optic networks using the step-index single-mode optical fiber SI-SMF and wavelength division multiplexing xWDM techniques.

NG-OFs open a new path in the field of spatial multiplexing in the photonic domain. In order to transfer spatial layered signals coming from the module of an 8element array it is proposed to use optical fiber TA-MCF, which includes nine single-mode cores (Fig. 1). a)

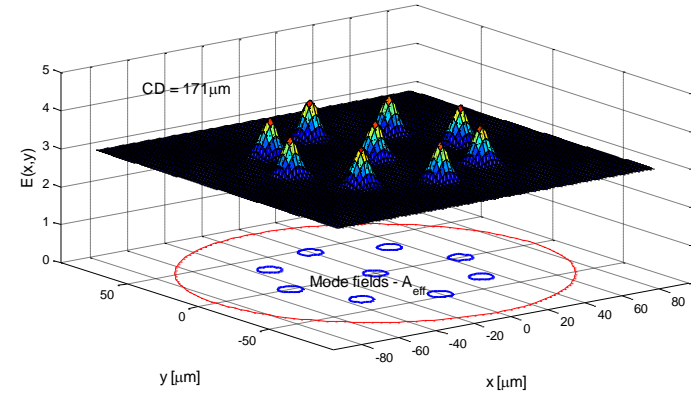

b)

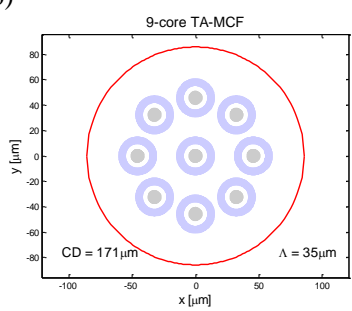

c)

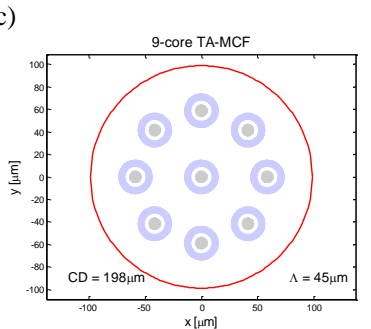

Fig. 1. Model of TA-MCF with changing CD and structure of 9 singlemode cores, each with a step-index profile: a) modal characteristic for $\mathrm{A}_{\mathrm{eff}}=90 \mu \mathrm{m}^{2}$ and $\left.\Lambda=35 \mu \mathrm{m}, \mathrm{b}\right)$ transverse profile for $\mathrm{A}_{\mathrm{eff}}=90 \mu \mathrm{m}^{2}$ and

$\Lambda=35 \mu \mathrm{m}, \mathrm{c})$ transverse profile for $\mathrm{A}_{\mathrm{eff}}=90 \mu \mathrm{m}^{2}$ and $\Lambda=45 \mu \mathrm{m}$.

In order to determine the parameters of the proposed nine-core TA-MCF (Fig. 1), we established the initial parameters: $\lambda=1550 \mathrm{~nm}, A_{\text {eff }}=90 \mu \mathrm{m}^{2}$ (obtaining this value of the effective mode field is possible with the right choice of the relative refractive indexes: $\Delta_{1}$ and $\Delta_{2}$, and the distances $r_{1}, r_{2}$, and $W$ : Fig. 2), where the essential elements that affect the value of $A_{\text {eff }}$ are $\Delta_{1}$ and $r_{1}$. The depressive trench parameters were set with respect to the radius $r_{1}$ of the single core: $r_{2} / r_{1}=2, W / r_{1}=1$.

*e-mail: zbizak@utp.edu.pl 


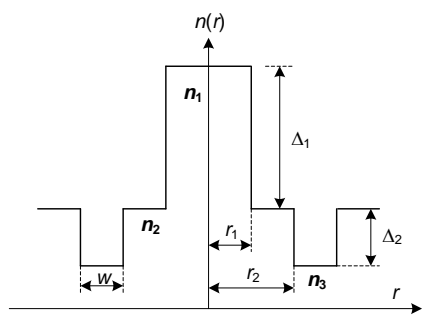

Fig. 2. Step-index profile for each TA-MCF core and its near the cladding trenched space.

From the characteristics of the cores with a surrounding depressive trench [Fig. 3 (b)] for the set of parameters defined above parameters and for $\Delta_{2}=-0.7 \%$, it was possible for the coupling coefficients $\kappa$ to be established (see Table 1).

Table 1 . Summary of readings on coupling coefficients $\kappa$ between the neighboring outer cores in TA-MCF (on the basis of [5])

\begin{tabular}{|c|c|c|c|}
\cline { 2 - 4 } \multicolumn{1}{c|}{} & \multicolumn{3}{c|}{ Coupling coefficient $\kappa\left[\mathrm{m}^{-1}\right]$} \\
\hline$A_{\text {eff }}\left[\mu \mathrm{m}^{2}\right]$ & $\Lambda=35 \mu \mathrm{m}$ & $\Lambda=40 \mu \mathrm{m}$ & $\Lambda=45 \mu \mathrm{m}$ \\
\hline 100 & $4 \times 10^{-3}$ & $3 \times 10^{-4}$ & $2.3 \times 10^{-5}$ \\
\hline 90 & $2 \times 10^{-3}$ & $1.3 \times 10^{-4}$ & $1.2 \times 10^{-5}$ \\
\hline 80 & $1 \times 10^{-3}$ & $6 \times 10^{-5}$ & $6 \times 10^{-6}$ \\
\hline
\end{tabular}

In the case of pitch $\Lambda=45 \mu \mathrm{m}$, there is an absolute need to increase the parameter CT (Cladding Thickness) of trenches with cores contained in the cladding space, which immediately increased the value of a standardized CD (Cladding Diameter). On the basis of a coupled-mode theory and the equivalent refractive index model [4], we can determine the mean value of statistical distribution of crosstalk $\mathrm{XT}_{\mathrm{m}}$, in a homogeneous multi-core fiber $\mathrm{MCF}$, with the formula [4-5]:

$$
\mathrm{XT}_{\mathrm{m}}=\frac{2 \kappa^{2} R L}{\beta \Lambda},
$$

where $\kappa$ is the coupling coefficient between neighboring cores, $\beta=2 \pi n_{\text {eff }} / \lambda$ - the propagation constant of each core, $R$ - the bending radius of fiber, and $L-$ the optical fiber length, respectively.

The density of the fiber cores in the MCF as a core multiplicity factor CMF can be determined by using the formula [6]:

$$
\mathrm{CMF}=\frac{n A_{\text {eff }}}{\pi(\mathrm{CD} / 2)^{2}},
$$

where $n$ is the core number in the MCF, and $A_{\text {eff }}$ is the effective modal area (field) of each core. $\mathrm{CMF}$ is the ratio of the effective surface of the guided light in the MCF and cross-section in the area of the cladding. With respect to the above sets, equations (1) and (2) have been used in order to draw the characteristics of the mean between-core crosstalk $\mathrm{XT}_{\mathrm{m}}$ as a function of optical path length and distance (pitch) between the neighboring outer cores, see Fig. 3. The resulting draws can be applied in the case of multi-core optical fibers TA-MCF with a ring arrangement of the outer cores and a step refractive index profile (relative to the previously mentioned parameters) - only for outer cores.

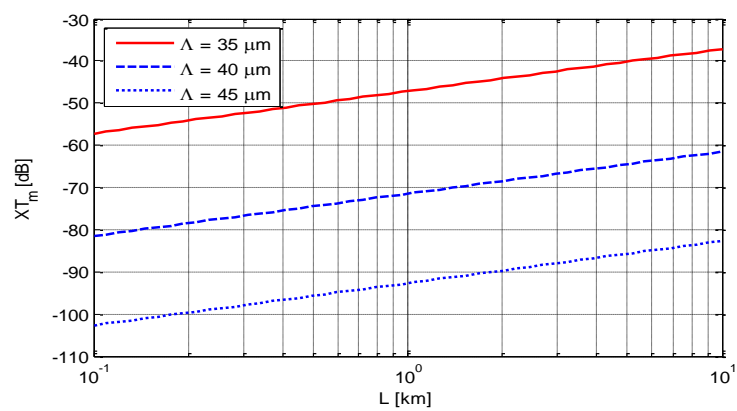

Fig. 3. The statistical mean values of neighboring crosstalk between outer cores in TA-MCF as a function of optical fiber length, for specified core pitches $\Lambda$.

Figure 4 shows indirectly the characteristics of the level of optical coupled-power from the outer cores to the inner core of TA-MCF, using the coupling coefficients $\kappa$ between neighboring cores [5], see Table 1.

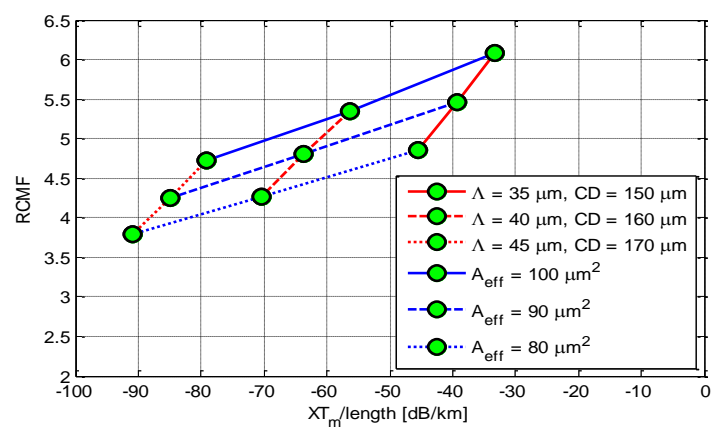

Fig. 4. Related Core Multiplicity Factor as a function of statistical mean crosstalk from outer cores to inner core in TA-MCF (on the basis of [5], see Table 1)

The formulas (1) and (2) were applied to calculate the above parameters. The calculations of the RCMF (Relative Core Multiplicity Factor) have been made with respect to the parameters of the standard single-mode single-core fiber with step index SI-SMF (ITU-T G.652D: $\mathrm{CD}=125 \mu \mathrm{m}, A_{\text {eff }}=80 \mu \mathrm{m}^{2}$ ), while the level of crosstalk by the unit of the fiber length has been made for the wavelength $\lambda=1550 \mathrm{~nm}$, the effective refractive index $n_{\text {eff }}=1.4675$ and the macro-bending radius $R=500 \mathrm{~mm}$.

The use of multi-core optical fibers TA-MCF in the connecting part between the base station and antenna units (Fig. 5), makes significant spatial multiplexing possible, not needing many fibers in a single cable. An example would be any antenna mast with an antenna array which has six sectors, where each antenna is an 8-element array. Spatial separation would require here, at least, a 48-fiber optical cable (not considering the fiber reserved for the antenna array control unit) of 1-core fibers. In the case of 
9-core fibers, this number drops to 6 - as many as the number of sectors around the mast, see Fig. 5.

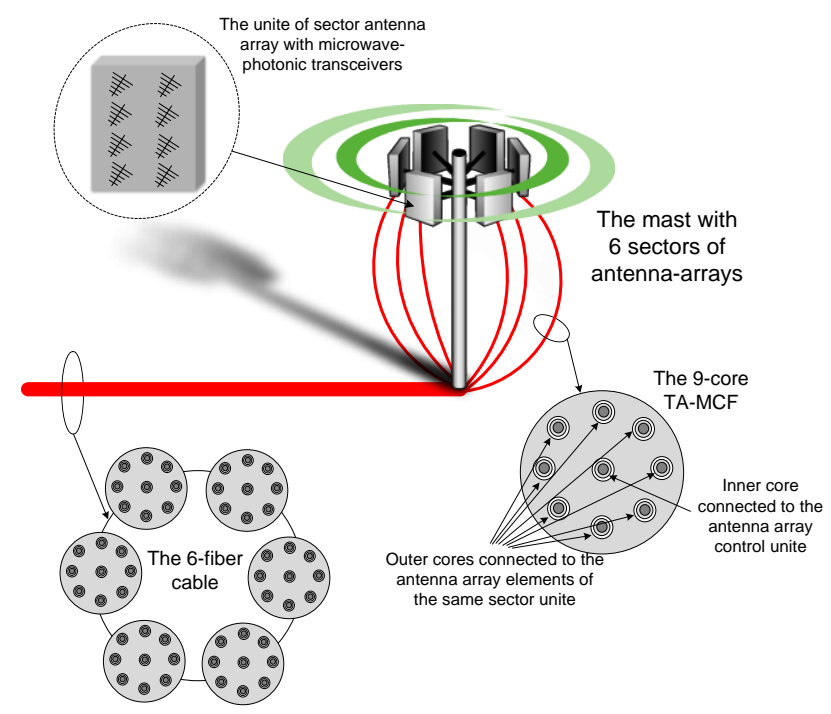

Fig. 5. The configuration idea of connections, near the mast, of the sector antenna array modules, which include bi-directional radiophotonic transceivers (modems), and the TA-MCF cable.

Figure 6 shows the assembling possibility of several configurations of the spatial channels connecting the mobile terminal to the base station eNodeB. It should be noted that the base station is a set of devices (radiophotonic modems, SDR processor, module for concentration of digital traffic, and intelligent module for the radio resource management). The eNodeB is connected, via TA-MCF modules, to the sector antenna arrays which enable wireless communication with mobile terminals.

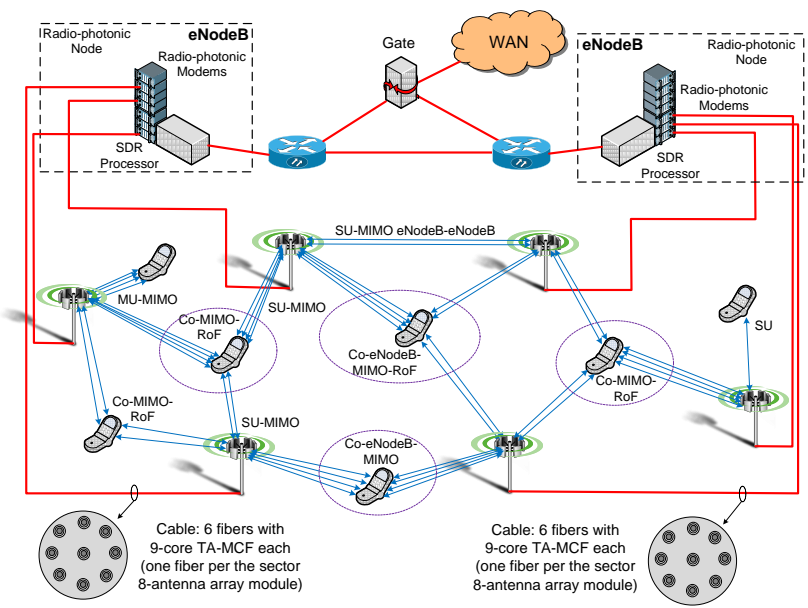

Fig. 6. Conception of a basic configuration network with distributed antenna array systems connected by multi-core banding-loss insensitive optical fibers TA-MCF.

RoF configuration allows the introduction of a different approach to the management of radio resources, since the base station may be equipped with a large number of antenna modules which are distributed over a large area. This enables to create better space paths separated from each other. A classical terminal equipped with a single antenna will be supported by diversity techniques, and the new generation of mobile equipment will use MIMO technology. For example, operating in the area of a single base station, from the eNodeB site, SU-MIMO (SingleUser MIMO) techniques may be used, where the sector supports a single user using space multiplexing and MUMIMO (Multi-User MIMO) in situations where more terminals are operating in a single sector, using the same frequency resources. Space multiplexing can be done in cooperation bewteen two or more base stations that communicate with each other through a core (cable) network or through a wireless interface. Such a technique known as Collaborative-MIMO (Co-eNodeB-MIMO) was first introduced in the LTE (Long Term Evolution). If the RoF distributed antenna system format is implemented, it is possible to introduce the technology that enables the interworking of more sector modules belonging to a single base station (suggested name: Co-MIMO-RoF) - Figure 6. When we combine the proposed technique Co-MIMORoF and well known from LTE network technology CoeNodeB-MIMO, we can get the hybrid technology [suggested name: Co-eNodeB-MIMO-RoF(CollaborativeeNodeB-Multiple Input Multiple Output-Radio over Fiber)], see Figure 6, which is due to the possibility of direct communication of the base stations through the antenna array modules and easy to implement.

\section{References}

[1] J. Cooper, Electron. Lett. 26(24), 2054 (1990).

[2] S. Randel, R. Ryf, A.H. Gnauck, M.A. Mestre, C. Schmidt, R.J. Essiambre, P.J. Winzer, R. Delbue, P. Pupalaikis, A. Sureka, Y. Sun, X. Jiang, R. Lingle, Mode-multiplexed 6x20-GBd QPSK transmission over 1200-km DGD compensated few-mode fiber, in Proc. OFC/NFOEC (2012).

[3] L. Maksymiuk, J. Siuzdak, Successful IEEE 802.11n 2-channel MIMO transmission over standard graded index multimode fiber in passband, 26th Biennial Symposium on Communications (QBSC), 15-18 (2012).

[4] T. Hayashi, T. Taru, O. Shimakawa, T. Sasaki, E. Sasaoka, Opt. Expr. 19(17), 16576 (2011).

[5] K. Saitoh, M. Koshiba, K. Takenaga, S. Matsuo, IEEE Phot. Tech. Lett. 24(21), 1898 (2012).

[6] K. Takenaga,Y. Arakawa, Y. Sasaki, S. Tanigawa, S.Matsuo, K.Saitoh, M. Koshiba, Opt. Expr. 19(26), B542 (2011). 